\title{
Inborn Errors of Metabolism and the Endocrinologist
}

\author{
Bashari WA, Oyibo SO \\ Department of Endocrinology, Peterborough City Hospital, Peterborough, UK
}

\begin{abstract}
Introduction
Inborn Errors of Metabolism (IEM) individually are rare but collectively are common. These are a group of disorders with single gene-enzyme defects which cause a clinically significant block in a metabolic pathway. Patients are best managed at metabolic disease specialist centres, where they have the benefit of a multidisciplinary team involving the metabolic disease specialist, dietitians and child psychologists.

These patients are living longer, migrating to areas far from their specialist care centres and can present with life-threatening problems to your hospital. We present two such cases.
\end{abstract}

\section{Case 1}

A 37 year old female with Ornithine Transcarbamylase Deficiency (OTCD), well-managed at a specialist care centre since childhood, but now relocated to our area. This X-linked recessive inability to convert ammonia to urea (urea cycle disorder) puts her at risk of lift-threatening hyperammonaemia if she gets infections or other stressful conditions. She has had several admissions, has an individualised care pathway for emergency treatment and for peri-operative management. She also has epilepsy and learning difficulties. She is on a low-protein diet and is prescribed long-term nitrogen-scavenger medications, which are not well documented in the British National Formulary for adults. She has regular outpatient follow-up with open-access for urgent plasma ammonia estimation if required.

\section{Case 2}

An 18 year old male with medium-chain acyl-CoA dehydrogenase deficiency (MCADD) well-managed at a specialist care centre since childhood but now shared care with our centre. This autosomal recessive inability to breakdown medium-chain fatty acids to provide energy during periods of low-calorie intake (infections, fasting or vomiting) puts him at risk of encephalopathy and sudden death from the accumulation of toxic fatty acids and hypoglycaemia. He has an individualised care pathway for emergency treatment and has reqular follow-up in the outpatient clinic.

\section{Why we don't remember IEM}

- Rare conditions

- Mainly affects children

- Well-looked after by Paediatricians and Specialists in Metabolic Medicine

- Attend Adult Metabolic Medicine clinics/MDT

- Not included in our SpR training

\section{Why we must remember IEM}

- With good specialist care (early diagnosis) more are surviving into adulthood

- Some patients migrate and find it difficult to attend specialist centres

- Patients may be brought to your hospital with an acute life-threatening emergency

- Other specialities will look to you for help

\section{How we overcome the phobia for IEM}

- Know your limitations

- Get previous correspondences, management protocols, etc.

- Be aware of patient's specialist centre

- Listen to parents/carers

- Liaise with specialist centre (dietitians, MDT), BIMDG

- Read about the condition (clinical features and prevention of crises)

- Emergency treatment protocols

- Non-emergency treatment protocols

\section{Conclusion}

Training concerning some Inborn Errors of Metabolism needs to be incorporated into endocrinology SpR training as more secondary care centres are caring for such patients. The importance of seeking advice from metabolic disease centres and the Inherited Metabolic Disease Group cannot be overemphasised. 